\title{
2.2 X-RAY OBSERVATIONS OF NP 0532
}

\author{
SAUL RAPPAPORT \\ Center for Space Research, Massachusetts Institute of Technology, \\ Cambridge, Mass., U.S.A.
}

The Crab Nebula pulsar, NP 0532, is the only one of the 56 known pulsars which has been observed to pulse in the optical spectrum. Even more spectacular are the vast quantities of pulsed X-rays which it has been found to emit. Soft X-rays in the 1-10 keV region have been observed during four sounding rocket flights (Fritz et al., 1969; Bradt et al., 1969; Boldt et al., 1969; Ducros et al. 1970) and harder X-rays, 25-200 keV, have been detected during two balloon flights (Fishman et al., 1969a, b; Floyd et al., 1969). From the results of these six experiments we summarize the properties of the X-ray pulsations from NP 0532.

The X-ray and optical pulse profiles for NP 0532 have striking similarities while a detailed comparison reveals several distinct differences. In X-rays the characteristic main pulse and interpulse are present, but unlike the optical case (Lynds et al., 1969; Warner et al., 1969; Papaliolios et al., 1970) the energy in the broader and smaller amplitude interpulse is about equal to that in the main pulse. The separation of the two pulses is $13.2 \pm 0.3 \mathrm{msec}$ (Bradt et al., 1969; Fritz et al., 1970; Rappaport et al., 1970). The full widths at half maximum of the main pulse and interpulse are $1.0 \pm 0.2$ msec (Fritz et al., 1970; Rappaport et al., 1970) and $2.8 \pm 0.3 \mathrm{msec}$ (Fritz et al., 1970; Rappaport et al., 1970) respectively. The interpulse has a slow rise and rapid decline while the opposite is true of the optical interpulse (Lynds et al., 1969; Warner et al., 1969; Papaliolios et al., 1970). In the $13 \mathrm{msec}$ interval between the two pulses the X-ray flux does not go to the dc background level but remains at about $10 \%$ of the peak rate in the main pulse (Bradt et al., 1969; Rappaport et al., 1970).

The MIT experiment of April 27, 1969 (Bradt et al., 1969) featured nearly simultaneous optical and X-ray observations of the Crab pulsar. The time of the main optical pulse as determined at Mount Palomar and McDonald observatories differs from the time of the main X-ray pulse by only $0.4 \pm 0.5 \mathrm{msec}$. Recently the Princeton group (Groth and Wilkinson, 1970) calculated from their data the arrival time of the main optical pulse at White Sands Missile Range nearest the time of the M.I.T. rocket launch. The time they obtain differs from the time of the main X-ray pulse by only $0.22 \pm 0.3 \mathrm{msec}$. It is now clear that the radio (corrected to infinite frequency) (Rankin et al., 1970), optical and X-ray pulses are all coincident to within $\sim 0.3 \mathrm{msec}$.

The time averaged flux of pulsed soft X-rays (1-10 keV) from NP 0532 is about $8 \%$ of the soft X-ray flux received from the Crab Nebula (Bradt et al., 1969). The $X$-ray flux from the nebula in this energy range is $3 \times 10^{-8} \mathrm{erg} \mathrm{cm}^{-2} \mathrm{sec}^{-1}$ (Kellogg, 1971) and the corresponding X-ray luminosity is $1.5 \times 10^{37} \mathrm{erg} \mathrm{sec}^{-1}$. The time averaged flux (1-10 keV) from the pulsar is $2.5 \times 10^{-9} \mathrm{erg} \mathrm{cm}^{-2} \mathrm{sec}^{-1}$ (Bradt et al., 1969) and its X-ray luminosity depends on the geometry of the pulsar beam. When the 
$\mathrm{X}$-ray flux from the pulsar is maximum it is about half as bright as the non-pulsed X-radiation from the Crab (Bradt et al., 1969; Rappaport et al., 1970). The pulsar puts out $\sim 100$ times as much energy in X-rays as in the optical (Oke, 1969) and $\sim 10^{5}$ times as much as in the radio region $\left(>10^{8} \mathrm{~Hz}\right.$ ) (Rankin et al., 1970; Comella et al., 1969).

The X-ray energy spectrum of the Crab Nebula in the region 1-560 keV can be well represented by a power law with spectral index $\alpha=1.1$ (Kellogg, 1971). The pulsed component in the range 2-200 keV decreases with decreasing energy from about $15 \%$ of the flux density of the Crab to $\sim 5 \%$ (Fritz et al., 1969; Bradt et al., 1969; Ducros et al., 1970; Fishman et al., 1969b; Floyd et al., 1969). The MIT measurement in the interval 1-1.5 keV (Rappaport et al., 1970) indicates that the pulsed component is only $3.5 \pm 1.2 \%$ of the nebular radiation at these energies. This apparent decrease in the pulsed radiation is not due to interstellar photoelectric absorption since the same energy X-rays from the nebula are not significantly attenuated (Rappaport et al., 1969). Scattering by dust grains in the interstellar medium has been suggested by Slysh (1969) but an earlier calculation (Overbeck, 1965) and more recent calculations (Naranan and Shah, 1969; Ryter, 1970; Bowyer et al., 1970) show the optical depth for X-ray scattering at $1 \mathrm{keV}$ for the Crab Nebula is small $(\sim 0.2)$. Absorption of the very soft $\mathrm{X}$-rays could be taking place in the immediate vicinity of the pulsar or the intrinsic spectrum could be changing at $1 \mathrm{keV}$.

\section{References}

Boldt, E. A., Desai, U. D., Holt, S. S., Serlemitsos, P. J., and Silverberg, R. F.: 1969, Nature 223, 280.

Bowyer, C. S., Mack, J., and Lampton, M.: 1970, Nature 225, 1125.

Bradt, H., Rappaport, S., Mayer, W., Nather, R. E., Warner, B., MacFarlane, M., and Kristian, J.: 1969, Nature 222, 728.

Comella, J. M., Craft, H. D., Lovelace, R. V. E., and Sutton, J. M.: 1969, Nature 221, 453.

Ducros, G., Ducros, R., Rocchia, R., and Tarrius, A.: 1970, Nature 227, 152.

Fishman, G. J., Harnden, F. R., Jr., and Haymes, R. C.: 1969a, Astrophys. J. Letters 156, L107.

Fishman, G. J., Harnden, F. R., Jr., Johnson, W. N., III, and Haymes, R. C.: 1969b, Astrophys. J. 158, L61.

Floyd, F. W., Glass, I. S., and Schnopper, H. W.: 1969, Nature 224, 50.

Fritz, G., Henry, R. C., Meekins, J. F., Chubb, T. A., and Friedman, H.: 1969, Science 164, 709.

Fritz, G., Henry, R. C., Meekins, J. F., Chubb, T. A., and Friedman, H.: 1971, preprint.

Groth, E. J. and Wilkinson, D. T.: 1970 (private communication).

Kellogg, E. M.: 1971, this symposium, Paper 1.5, p. 42.

Lynds, R., Maran, S. P., and Trumbo, D. E.: 1969, Astrophys. J., Letters 155, L121.

Naranan, S. and Shah, G. A.: 1969, Nature 225, 834.

Oke, J. B.: 1969, Astrophys. J. Letters 156, L49.

Overbeck, J.: 1965, Astrophys. J. 141, 864.

Papaliolios, C., Carleton, N. P., and Horowitz, P.: 1970, Nature 228, 445.

Rankin, J. M., Comella, J. M., Craft, H. D., Jr., Richards, D. W., Campbell, D. B., and Counselman, C. C., III: 1970, Astrophys. J. 162, 707.

Rappaport, S., Bradt, H. V., Mayer, W.: 1969, Astrophys. J. Letters 157, L21.

Rappaport, S., Bradt, H., and Mayer, W.: 1971, Nature 229, 40.

Ryter, Ch.: 1970, Nature 226, 1040.

Slysh, V. I.: 1969, Nature 224, 159.

Warner, B., Nather, R. E., and MacFarlane, M.: 1969, Nature 222, 233. 


\section{Discussion}

Sir Bernard Lovell: Is the ratio of pulsed to non-pulsed X-radiation a function of energy as indicated by the difference in the balloon and the rocket results or are there uncertainties in the two types of measurement?

S. Rappaport: It is a definite effect.

$N$. Visvanathan: In X-rays, the $8 \%$ pulsed intensity is compared to the total intensity from all over the Crab, while in optical the pulsed intensity is $18 \%$ compared to the $1 \mathrm{sec}$ arc Nebula around the pulsar.

J.P. Ostriker: Can you quote limits for the periodic component of the X-rays observed from the two other supernova remnants emitting X-rays?

E. Kellogg: Upper limits on pulsed X-ray emission, 1-10 keV, from Cas A and Tycho, assuming a single pulse occupying one tenth of a period, with periods between 8 and $35 \mathrm{msec}$, with $99 \%$ confidence are

$$
\begin{array}{ll}
\text { Cas A } & 15 \% \\
\text { Tycho } & 19 \%
\end{array}
$$

Results obtained by Lorenstein, Kellogg, and Gursky, are published in Astrophys. J. 160, (1970) 199.

J. Kristian: By how much is your comparison with the optical FWHM affected by the fact that the optical peak is still unresolved with time resolution as short as $20 \mu \mathrm{sec}$ ?

$P$. Horowitz: Even though the turn-around near the optical peak hasn't been resolved, the FWHM estimate is not much affected since the rise to maximum is approximately linear from both sides and therefore the height of the peak is probably not in error by more than the rise during one timing channel, i.e. less than $5 \%$ of the total height.

D. M. Jennings: Ducros et al. have recently reported two further inter-pulses, one following both the main pulse and the well-known' inter-pulse. Have you any information on these?

S. Rappaport: No.

F. G. Smith: There is another inter-pulse component observed at radio frequencies, which we will hear about later in a report by Mr. Schönhardt. 